\title{
Erratum to: The how and why of adult neurogenesis
}

\author{
Inmaculada Ortega-Perez • Kerren Murray • \\ Pierre-Marie Lledo
}

Published online: 29 June 2010

(C) Springer Science+Business Media B.V. 2010

\section{Erratum to: Hist (2007) 38:555-562 \\ DOI 10.1007/s10735-007-9114-5}

Unfortunately, in the section Abstract the sentence starting with

"Is adult neurogenesis an atavism...cell can detect?" should be read with quotation as shown below.

"Is adult neurogenesis an atavism, an empty-running leftover from evolution? What is adult neurogenesis good for and how does the brain 'know' that more neurons are needed? How is this functional demand translated into signals a precursor cell can detect?"

In the section Neurogenic permissiveness in the adult brain the sentence starting with

"The term neurogenic...olfactory system." should be read with quotation as shown below.

"The term neurogenic implies at least two things: first, the presence of immature precursor cells from which new neurons can develop, and second, a certain type of microenvironment that is permissive for neurogenesis to occur. In the adult mammalian brain, there are two known neurogenic regions: the dentate gyrus subgranular zone at the hippocampus (SGZ) and the SVZ/olfactory system"

In the section Neurogenic permissiveness in the adult brain the sentence starting with

The online version of the original article can be found under doi:10.1007/s10735-007-9114-5.

I. Ortega-Perez $(\square) \cdot$ K. Murray $\cdot$ P.-M. Lledo

Laboratory for Perception and Memory, Pasteur Institute, CNRS

URA 2182, 25 rue du Docteur Roux, Paris Cedex 15 75724,

France

e-mail: iortega@pasteur.fr
"We refer to the rest of the brain...or implanted precursor cells" should be read with quotation as shown below.

"We refer to the rest of the brain as non-neurogenic, although this categorization might ultimately turn out to be premature, and such terms as potential neurogenic or reactive neurogenic zones might be more appropriate (Fig. 2). There is still an ultimate test for qualifying a region as neurogenic: a neural precursor cell implanted in a neurogenic region should develop into a neuron and when grafted into a non-neurogenic region it should become a glial cell or die. Thus, the definition of 'neurogenicity' is based on a general and physiological neurogenic permissiveness rather than on the presence or absence of a neural precursor cell alone. The key questions become, then, what makes a brain region neurogenic? How is neurogenic permissiveness defined on a molecular and cellular level?

In sum, neurogenic regions are characterized by: (1) the presence of neural precursor cells, (2) the presence of a microenvironment consisting of cell-cell contacts and diffusible factors promoting neural development of the precursor cells and (3) a neurogenic potential that can be tested by the implantation of neural precursor cells into this region. Consequently, non-neurogenic regions may contain precursor cells, but lack distinctive germinative cell clusters as well as the permissive microenvironment that under physiological conditions would promote neurogenesis from local or implanted precursor cells."

In the section Neurogenic permissiveness in the adult brain the sentence starting with

"At the core of the concept...stem cell niche." should be read with quotation as shown below.

"At the core of the concept of neurogenic permissiveness is the assumption that precursor cells in the neurogenic zones are embedded into a microenvironment with 
which they form a functional unit, the so-called stem cell niche."

List the new references in Reference list as given below:

1. A central reference is missing in the article: OrtegaPerez I, Murray K, Lledo P-M (2007) The how and why of adult neurogenesis. J Mol Histol 38(Springer):
555-562 (Leading author: Lledo P-M ), in which the only participation of Murray $\mathrm{K}$ was English correction. This correction is published with the apologies of the director of the lab Lledo P-M, the sole and unique responsible of this mistake

2. The missing reference is: Kempermann G (2006) Adult neurogenesis. Oxford University Press 